\title{
Osteomie der Knöchelgabel \\ nach in Achsenabweichung verheilten Frakturen
}

\author{
D. Höntzsch
}

\section{Zusammenfassung}

Die Knöchelgabel bildet zusammen mit dem Talus das Scharniergelenk des oberen Sprunggelenkes. Es handelt sich um ein belastetes Gelenk, welches auf Veränderungen sehr empfindlich reagiert. Beschwerden und eine spätere fortschreitende Arthrose sind die gefürchteten Folgeschäden. Die Knöchelgabel setzt sich aus der Tibiagelenkfläche, der Vorder- und Hinterkante, dem Innen- und Außenknöchel sowie aus der straffen gelenkigen Syndesmose zusammen. Fehlheilungen können nach konservativer und operativer Behandlung auftreten. Um die richtige Korrekturosteotomie planen zu können, ist eine gute Röntgendiagnostik erforderlich. Das Behandlungsziel soll die Knöchelgabel möglichst anatomisch wieder herstellen. Dies wird erreicht, wenn die fehlverheilten Gabelanteile entsprechend osteotomiert und nach Korrektur übungs- und zumindest teilbelastungsstabil mit einer Osteosynthese versorgt werden. Bei Verlängerungsosteotomien am Außenknöchel müssen entsprechende additive Interponate aus einem autologen Spongiosablock eingesetzt werden. Für die Osteosynthesen werden neben Zuggurtung, Kleinfragmentschrauben und Platten verwendet. Oberhalb der Syndesmose sollten 3,5 mm DCP oder LC-DCP gegenüber den schwächeren Drittel. Rohrplatten bevorzugt werden. Für die Nachbehandlung sollte Übungs- und Teilbelastungsstabilität bestehen. Eine gezielt eingesetzte Physiotherapie muss die operative Behandlung ergänzen.

\section{Einleitung}

Sprunggelenksfrakturen zählen zu den häufigsten Verletzungen. Die Versorgung kann in den meisten Fällen einfach und erfolgreich, aber in vielen Fällen auch sehr anspruchsvoll sein.

Die Knöchelgabel bildet zusammen mit dem Talus (Sprungbein) das Scharniergelenk des oberen Sprunggelenkes [4]. Wie alle Gelenke, besonders der unteren belasteten unteren Extremität, reagiert dies auf Unregelmäßigkeiten, Stufen und Kanten in den Gelenkflächen sowie der Gelenkführung (Gelenkgabel) empfindlich $[1,5]$. Die Folgen können Bewegungseinschränkung, akute frühe Schmerzen, chronische späte Schmerzen und eine fortschreitende Arthrose sein. Um diese Folgen zu vermeiden, gelten für die Versorgung des Sprunggelenkes und insbesondere der Sprunggelenksgabel weiterhin die Prinzipien, dass möglichst anatomisch rekonstruiert werden soll, bzw. dass Fehlstellungen nach Verheilung möglichst bald und gut ausgeglichen werden müssen [5]. Beim Sprunggelenk handelt es sich um ein Gelenk, welches auf Veränderungen sehr empfindlich, d.h. nicht tolerant reagiert. Um so wichtiger ist die Wiederherstellung der anatomisch korrekten Situation.

\section{Anatomie (Abb.1)}

Die Knöchelgabel setzt sich aus folgenden Elementen zusammen:

- Tibiafläche, konkav gewölbt mit

- Vorder-/Hinterkante (bei Fraktur = Volkmann'sches Dreieck

- Innenknöchel

- Außenknöchel

- Gelenks- und Bandverbindung zwischen Außenknöchel und Talus (Syndesmose).
Dieses „Quartett“ bildet eine „Gelenkpfanne“ für den Talus (Sprungbein). Das Sprungbein selbst ist ein trapezförmiger Kubus, hinten schmaler als vorne $[3,4]$ (Abb.1).

Der Gelenkteil zum Unterschenkel ist eine entsprechend leicht asymmetrische halbe Rolle, welche nach proximal zeigt.

Diese Halbrolle läuft in der entsprechend geformten Tibiafläche und wird durch Innen- und Außenknöchel („Knöchelgabel“) geführt, so dass die Scharnierbewegung des oberen Sprunggelenkes möglich ist.

Die Anatomie so genau zu erklären ist wichtig, um die Bedeutung der einzelnen Elemente zu verstehen. Letztendlich kann jedes Einzelelement nach einer Fraktur fehlverheilen, wenn das Behandlungsziel, sei es operativ oder konservativ, nicht erreicht worden ist.

\section{Fehlstellungen}

Mögliche Fehlstellung der Knöchelgabel:

Tibiafläche:

Stufe in der Gelenkfläche

Vorderkante disloziert

Hinterkante disloziert

Innenknöchel:

zu kurz/zu lang

abstehend, d.h.

varisch $=$ Knöchelgabel zu weit anstehend, d.h.

valgisch $=$ Knöchelgabel zu eng

Syndesmose: zu weit/zu eng

Außenknöchel:

zu kurz/zu lang

gedreht

varisch, d.h. Knöchelgabel zu eng valgisch, d. h. Knöchelgabel zu weit kombinierte Fehlstellungen 

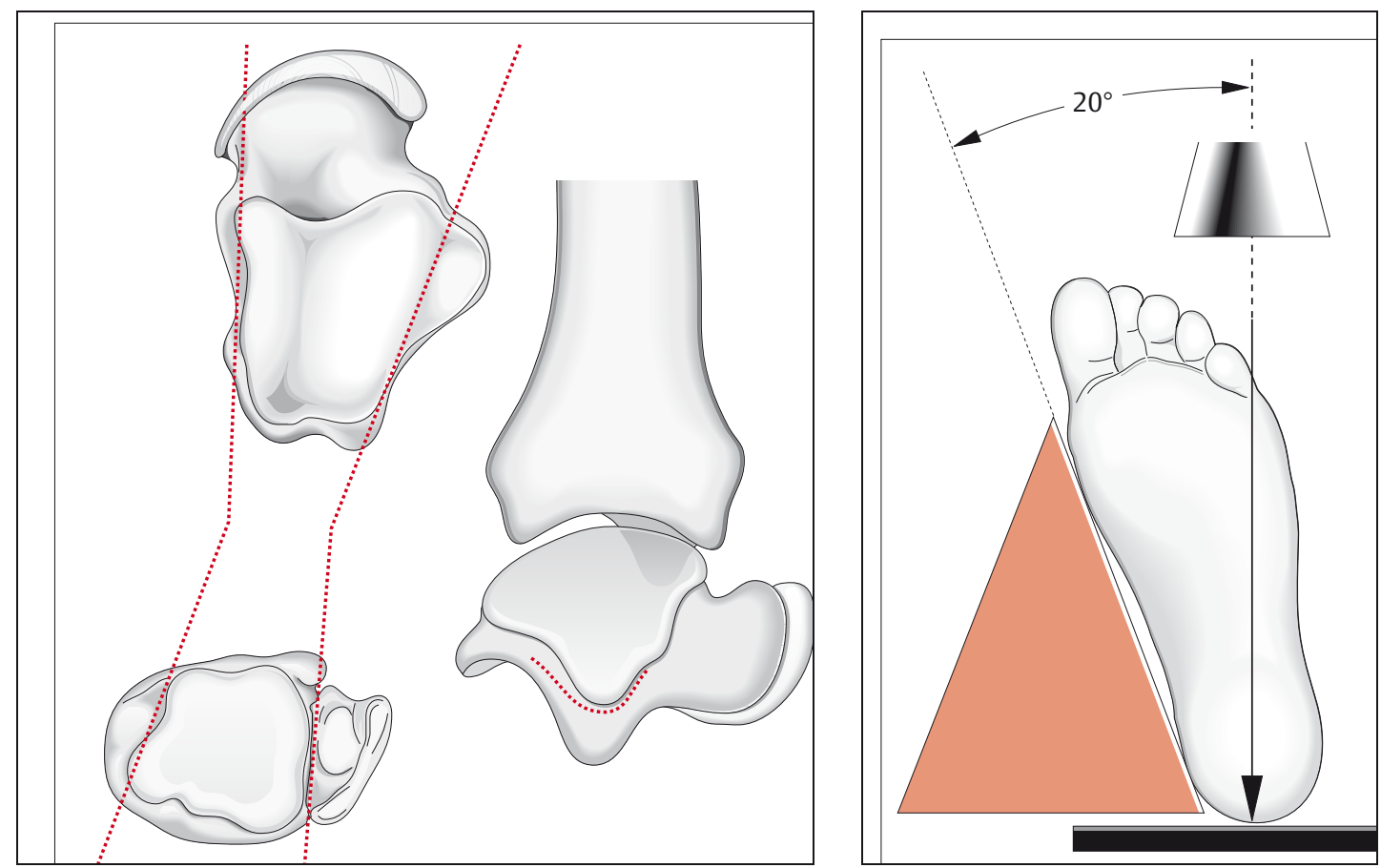

Abb. 2 (nach $\mathrm{AO}$ Manual [2]): Das Sprunggelenk muss bei der a.p.-Röntgenaufnahme $20^{\circ}$ nach innen gekippt werden, um so einen möglichst guten Einblick in den Gelenkspalt zwischen Talus und Innenknöchel und Talus und Außenknöchel zu erreichen.

Abb.1 (nach Schauwecker [3]): Die Sprunggelenksgabel wird neben dem „Zwischenstück“ Tibiagelenkfläche vom Außen- und Innenknöchel gebildet. Dies stellt die „Gelenkpfanne“ dar, in welcher sich das Sprungbein scharnierartig bewegt. Eine Besonderheit liegt darin, dass dieses Sprungbein ein trapezförmiger Kubus, hinten schmaler als vorne ist. Primäroperation oder die hier angesprochenen Korrekturoperationen müssen die genaue Einstellung der Gabel wieder herstellen, um eine zu lockere oder eine zu straffe Führung zu vermeiden.

\section{Diagnostische Maßnahmen}

Um Fehlstellungen am Sprunggelenk genau beurteilen zu können, sind im Regelfall Aufnahmen des Sprunggelenkes in 2 Ebenen ausreichend. Es muss aber auf Grund der komplexen und definierten Gabelstellung erreicht werden, dass der Einblick durch die Röntgenaufnahmen in genau korrektem und definiertem Strahlengang erfolgt, und dass die Bilder gut zentriert und belichtet sind $[1,2,3]$ !

Für den seitlichen Strahlengang bedeutet dies, dass die „Kuppel“ des Talus und der Sprunggelenksspalt in seiner Sichelform genau getroffen wird. D.h. der Sprunggelenksspalt muss ohne Überlagerung und der Talus ohne Doppelkontur und abgewandte Taluskante abgebildet sein. Für den a.p.-Strahlengang gilt, dass die knöcherne Struktur, welche die Gabel bildet und die Gelenksspalte eingesehen werden können. Im Regelfall wird dies durch eine $20^{\circ}$ ige Innenrotation erreicht (Abb. 2). Nur so lassen sich die Gelenk- spalte zwischen Talus und lnnenknöchel, zwischen Talus und kaudaler Tibiafläche, die „Jammerecke“ laterale Taluskante Außenknöchel/laterale Taluskante und der Gelenkspalt zwischen Außenknöchelfläche zu Talusaußenrand beurteilen.

Schon diese Auflistung zeigt, dass mehrere Gelenkpartien exakt dargestellt werden müssen. Beim gesunden oder wenig verformten Sprunggelenk ist dies durch eine exakte Einhaltung der Vorgaben möglich. Bei komplexen Sprunggelenksveränderungen oder komplizierten Fehlverheilungen kann es sein, dass die Fehlstellungen nicht mit einer Aufnahme dargestellt werden können. Hier kann es erforderlich sein, Zusatzaufnahmen wie Schrägaufnahmen oder unter kontinuierlicher Röntgenkontrolle angefertigte Aufnahmen im „besten“ Strahlengang anzufertigen. In Ausnahmefällen kann ein CT weiterhelfen. In Expertenhand hat sich in Einzelfällen auch eine präoperative $\mathrm{Ar}$ throskopie bewährt.
Hinweise zur Röntendiagnostik:

- streng seitliche Aufnahmen

a. p.-Aufnahmen $20^{\circ}$ innengedreht

- evtl. individuelle Einstellung für die Sprunggelenksspalte von vorne

bei komplexen Veränderungen und bei Defiziten nach den Normal-Röntgenaufnahmen $\rightarrow \mathrm{CT}$.

Vor der radiologischen Untersuchung und unabdingbar ist eine eingehende klinische Untersuchung der Funktion und Form des oberen und unteren Sprunggelenkes sowie des Fußes. Dies sollte durch die Beurteilung des Gangbildes und von Funktionsstellungen wie Zehenspitzenstand, Hackenstand und Gangbild barfuß und in Schuhen ergänzt werden. Des Weiteren ist die genaue Anamneseerhebung und das derzeitige Beschwerdebild aufzunehmen. Wie immer spielen natürlich Gesamtzustand, Neben- und Grunderkrankungen, Alter, Beruf und berufliche Anforderungen sowie das subjektive Beschwerde- und Anspruchsbild eine wesentliche Rolle.

Auch ist wichtig zu beurteilen, ob durch die fehlverheilte Stellung bereits ein Sekundärschaden eingetreten ist, und ob sich dieser aufhalten oder umkehren lässt. 


\section{Behandlungsziel}

Für das Behandlungsziel einer Korrektur nach fehlverheilter Knöchelgabel sollte folgendes gelten:

- Wiederherstellung oder Verbesserung der Funktion

- Beschwerdelinderung

- Verbesserung der Prognose

Vermeidung einer noch nicht bestehenden Arthrose

- Eindämmung einer bereits bestehenden Arthrose

- Verbesserung der Dauerbelastbarkeit.

Bei eingetretener Fehlheilung im Bereich der Knöchelgabel werden diese Ziele am besten dann erreicht, wenn die Korrektur möglichst frühzeitig und mit einem möglichst anatomiegerechten Ergebnis durchgeführt wird.

\section{Operations- und Korrekturmöglichkeiten}

a) Stufen in der Vorder- und Hinterkante der Tibiafläche

Diese Stufen können additiv oder subtraktiv sein. Wenn diese Stufe funktionell stört, Beschwerden verursacht und prog- nostisch ungünstig ist (ab 1/5 der Gelenkfläche), sollte die Frakturlinie, welche zu der Stufe führt, aufgelöst werden und das so neu gewonnene „Fragment“ im Sinne der richtigen Korrektur so manipuliert werden, dass die Gelenkfläche seine physiologische konkave Form wieder einnimmt. Die Schwierigkeit besteht in diesem Fall in der Auflösung der Frakturlinie und in dem Einstellen in der neuen korrekten Position. Die Stabilisierung kann dann mit Zugschrauben erfolgen. Es ist vorteilhaft, wenn Schraubenkopf und Gleitloch im verschobenen Fragment liegen. Im umgedrehten Fall ist der gewindetragende und damit zugaufbringende Anteil im Korrekturfragment im Regelfall und erfahrungsgemäß zu klein (Abb.3). Dies bedeutet für Korrekturen der Vorderkante einen Zugang von vorne oder seitlich vorne und für Korrekturen der Hinterkante einen Zugang von hinten seitlich. Je nach Hauptlokalisation hinter dem Innen- oder Außenknöchel.

\section{b) Innenknöchel}

Eine isolierte Fehlverheilung im Bereich des lnnenknöchels ist eher selten und kann im Einzelfall auch einmal symptom- los sein. Dies ist dann eine der wenigen Fehlstellungen, die fallbezogen auch belassen werden können. Eine Fehlverheilung im Bereich des lnnenknöchels kann im weiteren Sinn auch darin gesehen werden, dass bei Mitbeteiligung des Außenknöchels der Gelenkspalt zum lnnenknöchel durch eingeschlagene Bänder, Gewebepannus oder durch Kallus aufgefüllt ist. In einem solchen Fall ist eine Korrektur am Außenknöchel und Syndesmosenbereich ohne Korrektur am Innenknöchel nicht möglich. In diesem Fall muss zuvor der Innenknöchelbereich debridiert und somit befreit werden.

Für knöcherne Fehlheilung gilt ebenfalls, dass entweder die ehemalige Frakturlinie oder durch eine geeignete Osteotomie der Innenknöchel letztendlich in seine normale Stellung gebracht werden muss. Dies stellt den anspruchsvolleren Teil der Operation dar. Die Stabilisierung kann dann je nach Frakturlinie entweder mit einer Zuggurtung oder mit einer $\mathrm{Ab}$ stützplatte von medial erfolgen. Die Zuggurtung wiederum kann durch eine isolierte Zugschraubenosteosynthese, durch eine Zuggurtung oder durch eine Kombination aus beidem durchgeführt werden.

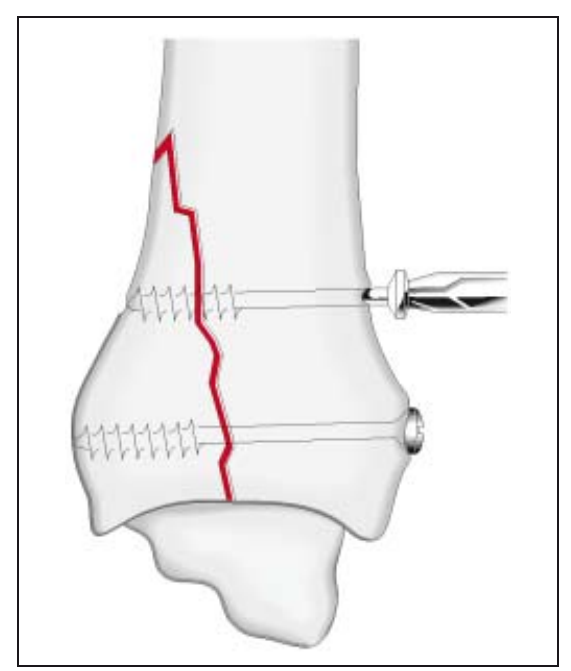

Abb. 3 (nach $\mathrm{AO}$ Manual [2]): Zugschrauben sollten so ausgerichtet sein, dass das Gleitloch im und der Schraubenkopf am kleineren Kantenfragment liegt. Nur so können die interfragmentären Druckkräfte durch Zug gegen das gut platzierte Gewinde aufgebracht werden.

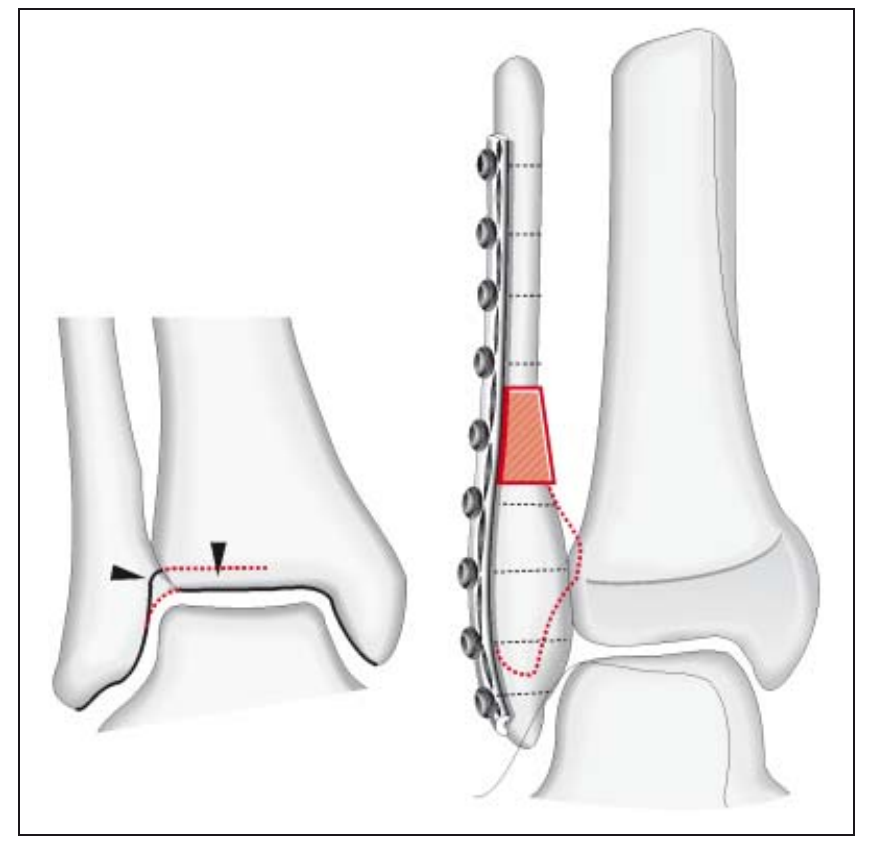

Abb.4 (nach AO Manual [2] und Schauwecker [3]): Eine der wichtigsten und notwendigsten Korrekturosteotomien an der Knöchelgabel stellen eine Osteotomie und korrekte Einstellung des Außenknöchels dar. Verlängerungsosteotomien mit Interposition eines Beckenkammspans sowie Stabilisierung mit einer Drittelrohrplatte oder 3,5-mm-DCP oder LC-DGP stellen bei zu kurz verheiltem Außenknöche eine sehr wertvolle Operation dar. Isoliert oder in Kombination mit der Verlängerung können so auch Rotation und Aufhebung einer Valgusfehlstellung korrigiert und stabilisiert werden. 

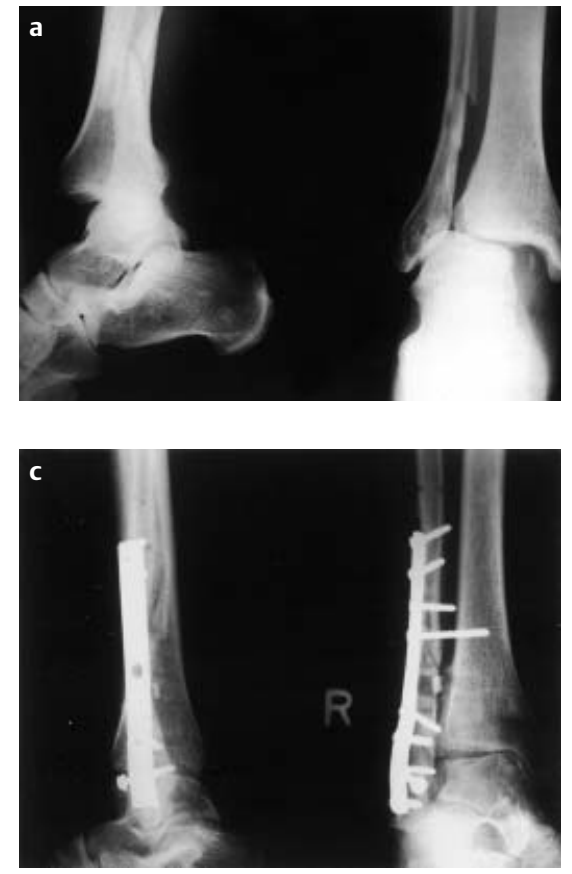

Abb.5a-d (ein Beispiel für die Fibulaverlängerung entsprechend Abb.4): (a) Fraktur mit Außenknöchelfraktur Typ Weber C, d. h. oberhalb der Syndesmose, Volkmannsches Dreieck und Dislokation nach dorsal sowie weite Sprengung im Bereich des Innenknöchelbereiches. (b) Ausheilung in leichter Verkürzung, Verdrehung des Außenknöchels, zu weite Syndesmose und sehr weiter innerer Gelenkspalt mit Pannusgewebe. (c) Operative Versorgung mit Verlängerung und Torsion des Außenknöchels. Es war eine leichte Überkorrektur notwendig, um das Sprungbein am Innenknöchel zu halten. Hierfür mußte der innere Gelenkspalt zwischen Innenknöchel und Talus revidiert und ausgeräumt werden. Die Syndesmose wurde temporär mit einer Stellschraube gehalten. (d) Ausheilungsbild 4 Jahre später mit guter Einstellung der Sprunggelenksgabel.

\section{c) Außenknöchel- und Syndesmosenbereich}

Außenknöchel- und Syndesmosenbereich sind im Regelfall eng vergesellschaftet und beeinflussen sich gegenseitig.

Je nach Fehlverheilung muss entweder die ehemalige Frakturlinie aufgelöst oder eine neue Osteotomie gesetzt werden. Häufig kann diese Korrektur proximal der Syndesmose durchgeführt werden. Die häufigsten Korrekturnotwendigkeiten sind folgende:
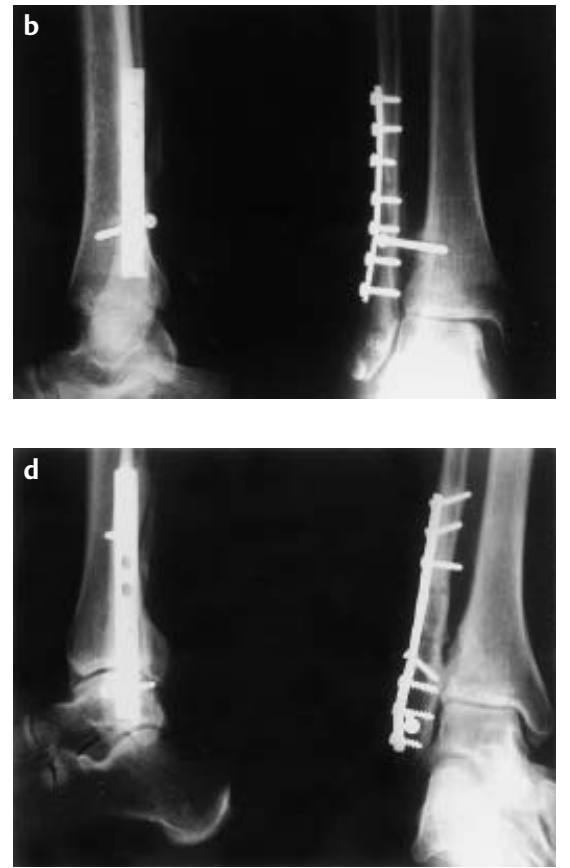

Leichte Überkorrektur des Außenknöchels in der Länge, deshalb an der Außenknöchelspitze postarthrotische Veränderungen. Spitzzipflige arthrotische Veränderungen im Bereich der Hinterkante. Das klinische Bild zeigt eine mäßige Einschränkung im oberen Sprunggelenk (10-0-30 nach der Neutral-0-Methode). Die volle Belastbarkeit ist möglich. Länger andauernde Maximalbelastungen verursachen dann Schmerzen. Die Röntgenreihe zeigt, wie die anfängliche Fehlstellung durch eine Korrektur, allerdings mit einer notwendigen leichten Überkorrektur nachhaltig beseitigt werden kann, zeigt aber auch, dass ein geschädigtes und nachoperiertes Sprunggelenk mit einer Arthrose reagiert. Allerdings wäre die im Zustand der Fehlverheilung (Bild b) in viel stärkerem Maße zu erwarten gewesen.

Häufigste Korrekturnotwendigkeit am Außenknöchel-/Syndesmosenbereich:

- Verlängerungsosteotomie (Abb.4)

- Rotation in die korrekte Stellung

- Aufheben einer Valgusstellung

- Wiederherstellung einer engen, gut geführten Syndesmose (nicht zu eng, evtl. „Befreiung“ des Innenknöchelspaltes - siehe oben)

- Kombinationen

Der Zugang am Außenknöchel ist im Regelfall direkt. Bei geplanter Revision der Syndesmose kann der Schnitt etwas nach ventral gelegt werden. Für allfällige Verlängerungen haben sich entsprechende Spongiosablöcke mit evtl. einem Kortikalisanteil aus dem Beckenkamm bewährt (Abb.5).

Zur Stabilisierung sollten im Bereich der Syndesmose und unterhalb der Syndesmose Drittelrohrplatten Anwendung finden. Oberhalb der Syndesmose sind bei Korrekturoperationen, nicht zuletzt wegen der auftretenden Kräfte 3,5-mmDCP oder LC-DCP zu bevorzugen $[2,3]$. Eine Revision und Neueinstellung der Syndesmose sollte temporär 6-8 Wochen mit einer Stellschraube stabilisiert werden. Je nach Dimension und Kräftigkeit des Skelettes sind entweder 3,5-mm-Schrauben oder in Einzelfällen auch 4,5-mm-Schrauben angezeigt.

\section{Nachbehandlung}

Die Stabilisierungsmethoden nach einer Korrektur sollte eine frühfunktionelle Nachbehandlung und eine Teilbelastung von $20 \mathrm{~kg}$ ermöglichen. Dies sind Anforderungen, die im Grunde genommen jede Osteosynthese und Korrekturoperation erfüllen sollten. Im Bereich des Sprunggelenkes ist dies aus Gründen einer guten Heilung, Verhinderung von Dystrophie und Erhalt bzw. Verbesserung der Funktion besonders hervorzuheben.

\section{Literatur}

${ }^{1}$ Heim U, Pfeiffer KM. Periphere Osteosynthesen. Springer, Berlin 1972

2 Müller ME, Allgöwer M, Schneider R, Willeneger $\mathrm{H}$. Manual der Osteosynthese AOTechnik. Springer, Berlin 1992

${ }^{3}$ Schauwecker F. Osteosynthesepraxis. Thieme, Stuttgart, New York 1981

${ }^{4}$ Waldeyer A. Anatomie des Menschen. de Gruyter, Berlin 1967

Prof. Dr. med. D. Höntzsch

Leitender Arzt

BG-Unfallklinik Tübingen

Schnarrenbergstr. 95

72076 Tübingen 